\title{
SCIDR: A Scalable Cluster based Inter-Domain Routing Protocol for Heterogeneous MANET
}

\author{
Rekha B \\ Research Scholar, \\ Department of Computer Science and Engineering \\ Jain University, Bangalore, India
}

\author{
D V Ashoka \\ Professor and Head \\ Department of Computer Science and Engineering, \\ JSSATE, Bangalore, India, Affiliated to VTU
}

\begin{abstract}
The area of Mobile Ad-hoc Network (MANET) has already been a topic of attention from past decade among the research community owing to its potential communication advantages as well as issues associated with it. However, the cases of inter-domain routing in the MANET have challenges furthermore compared to conventional MANET system. Border gateway protocol cannot be applied to support interdomain routing in mobile ad-hoc network as it cannot support the dynamic behavior of MANET. Hence, the this paper proposes a novel technique called as SCIDR-Scalable Cluster based Inter-domain Routing that is meant exclusively for heterogeneous MANET system. SCIDR is designed on a totally different principle compared to standard CIDR protocol, where CSI-Channel State Information, as well as channel correlation factor, are introduced to leverage further outcomes. For the first time, extensive performance parameters are used to benchmark the proposed system that ensures effective scalability.
\end{abstract}

\section{Keywords}

Clustering, Cluster based Inter-Domain Routing, Channel State Information, Channel Correlation, Optical Channel Gain.

\section{INTRODUCTION}

MANET comprises of interconnected nodes that are in mobility mode and hence it always exhibits dynamic topology behavior [1]. Owing to this dynamic topology, various problems shoot up e.g. routing issues, security issues, QoS issues, etc. [2]. Due to its infrastructure-less pattern, MANET has already found its identity in various applications like emergency operation, military applications, vehicular communications, etc. However, there is a different aspect of MANET that is called as heterogeneous MANET, where there are multiple types of mobilel nodes and multiple domains are interconnected with each other with an aid of network gateways. Border gateway protocol [3] could not assist to overcome the communication system for heterogeneous MANET owing to the dynamicity of MANET. In MANET, there is a consistent change in the connectivity of the network for which reason the inter-domain routing protocol will need to cater up the challenges of network partition as well as connectivity alterations. Moreover, there is no obvious boundary that segregates network domain and in the majority of the cases the multiple domains may overlap in the same geographic region [4]. It was also seen that the environment of MANET has been spontaneously tested with various routing protocols like AODV, DSDV, DSR, OLSR, etc., which have the capability to mitigate the dynamic topology. However, none of the existing routing protocols (reactive, proactive, geo cast, etc.) meet the challenges imposed by inter-domain routing in heterogeneous MANETs. After observing various significant studies done in the past, few of the studies have considered certain issues about the interoperability as well as scalability of the multiple networks with very less technical relevancy for supporting inter-domain routing in heterogeneous MANETs. The majority of the existing solution is related to cluster based networking that mainly targets to formulate a backbone of routing among the cluster heads. Such techniques are mainly meant to address scalability issues while performing inter-domain routing. This paper has considered one such standard work that has a mechanism, i.e., cluster based inter-domain routing in (MANET). Scalability is critical issues in large scale MANETs that requires to be addressed to ensure better performance. Hence, the proposed work presents a novel routing protocol that is built on the top of cluster based interdomain routing to address the scalability issues. Section 2 discusses the existing literature work that and the techniques used for overcoming issues of inter-domain routing, Section 3 discusses the proposed system and its significant characteristics followed by Section 4 that highlights the design principles adopted to implement SCIDR. Section 5 discusses the performance parameters adopted for the study highlighting the causes and implications of using it. Section 6 discusses the individual and comparative performance outcomes of SCIDR explicitly. Finally, Section 7 summarizes the paper.

\section{RELATED WORK}

Rekha B and D V Ashoka [5] presented a Minimal Gateway choice process to interconnect the MANETs, which can be utility to favor the "geo-based and inter-domain routing protocol". For gateway choice, the metrics like node density and adjoining nodes are respect. Two other metrics - number of hop and "least- load" path are utilizes for collections data transmission from a origin node in one domain to a target node of the different domain.

Chuah and Yang [6] described two inter-domain routing protocols for disruption tolerant networks, namely the gateway-based and the ferry-based approaches. Then, they demonstrated via resemblance studies that both inter domain routing schemes contribute correct delivery performance than the flat routing approach when separate groups are segregated from one another. They also show that the ferry based scheme execute higher delivery ratio and lower average end-to-end delay for the inter-domain traffic than Gateway-based interdomain routing (GBIR) when the ferry speed is higher than the normal node speed. Also, they demonstrated that the selection of intra-domain routing scheme affects the delivery achievement of intergroup messages. Using a multi hop intradomain routing scheme is correct than using a two-hop relay routing scheme. Subsequently, they rate how mobility models, 
some assembly affect the delivery achievement of the ferrybased scheme.

Dressler and Gerla [7] discussed the faces occurring from deploying effective synchronize for routing (to a exacting origin ID or resources or to indexed information) in mobile networks in multiple-domain network situation. They expand a solution to organization data with a spread hash table scheme. Based on their virtual cord protocol, using appropriate indirections they can implement inter-domain routing. That approach, though was still limited in detection efficient routes more multiple transit networks.

Dressler et al. [8] studied the capabilities of a bio-inspired routing heuristic, the Ant Colony Optimization approach, for inter-domain routing in essential coordinate-depend network environments. They first established a generalized routing framework that can maintain information about interconnected domains. In particular, the framework provides a microscopic view on gateways directly connecting neighboring domains and a macroscopic view on the highlevel domain topology.

Okundaye [9] proposed a routing solution that utilizes OSPF as a scalable routing result for an inter-domain network since it has variants that can work in both the fixed and MANET environment. They tested OSPF-MDR, a variant of OSPFv3 for MANETS and showed that after 20 minutes, the network of 200 routers running OSPF-MDR was seen to converge with all routers finding the shortest valid route to all other routers in the network

Comarela et al. [10] presented a new way to study the Internet at the AS level. They introduced a new measure, TRSD, allowing us to characterize the rate of change in the interdomain routing system over long periods of time.

Kaur et al. [11] proposed and evaluated two mechanisms SSR, Buzzer, which helps in securing inter-domain routing. Both mechanisms achieve the following goals:

They allow the coordination of the ASes so that they can have more exposure to path diversity. Help non-participating ASes to select valid routes. As a small group provides more security, many other Ases tries to join the secure group. Elmokashfi et al. [12] addressed the lack of a extensive toolbox to resemble BGP. It intends an exile topology generator that generates AS-level graphs that are annotated with profession relationships. The another component of the toolbox is a light-weight BGP simulator that is effective of capturing routing dynamics while scaling to network dimension of thousands of nodes. They employ their framework to search a set of what-if doubt regarding the impact of other topology parameters on BGP dynamics.

Yuanling et al. [13] revealed and analyzed the attack modes that the inter-domain routing method may suffer in the IPv6 environment. BGP4+ is an important inter-domain routing protocol of the next generation Internet.

Pan et al. [14] have tried to fill a gap between the designs of New Internet Architecture (NIA) and the evaluation efforts through an AS-level inter-domain routing system evaluation. The major idea was systematically defining a series of quantitative metrics to reveal hidden information and observations that may be useful in improving the status and deploying candidate new architectural solutions.

Wang et al. [15] proposed software defined Inter-domain Routing Plane (SRP). It can support flexible inter-domain routing policy, and create new business relationships between
Ases, based on multiple match fields of IP header. It also provides forwarding path diagnosis and interface between routing service and customers. They discuss its incremental deployment and challenges.

He et al. [16] proposed Hierarchy-Based Reduction (HBR), a network sampling method, which produces topologies that preserve the fundamental properties of the Internet graph, including, in particular, its hierarchical structure. Their approach provides a long-term solution to the difficult problem of AS-level routing evaluations: it can be used to generate small, realistic topologies in the future, starting from any newer or more complete Internet instance.

Lee et al. [17] presented a novel Inter- mobile ad-hoc network (MANET) Routing protocol describes Inter MR that may grip the heterogeneity and dynamics of MANETs. Their primary donation is an Inter-MANET address method depend on a diversity of node attributes (e.g., emblematic name, property, etc.); this permits dynamic merging/split of network topologies without a split Name Server.

Javed et al. [18] demonstrate that Poi-Root is highly accurate, works well even with partial information, and narrows down the cause to a single network or two neighboring ones. On controlled experiments, Poi Root is $100 \%$ accurate, as opposed to prior work that is accurate only $61.7 \%$ of the time.

\section{PROPOSED SYSTEM}

In order to design a novel cluster based inter-domain routing for mitigating scalability problems, the study was aggravated by the work completed by Zhou et al. by the title "Clusterdepend Inter-domain Routing" (CIDR) Protocol for MANETs", which was published in IEEE-2009 [19] Following are objectives to accomplish it:

To design an interior gateway protocol for heterogeneous MANET. To address the scalability issues. Motivated by this work, the proposed scheme SCIDR (Scalable-CIDR) is mainly targeted for performing cluster based inter-domain routing in heterogeneous MANET primarily emphasizing on the scalability issues. SCIDR illustrates the approach that permits a particular ad-hoc network to scale across heterogeneous link layers. The ultimate objective of this approach to have a notion of internet, i.e., network of networks, thus an analogy of MANET-internet. This technique enables integrating the MANET into the hierarchical internet and sustains the immigration of the mobile nodes from the internet into and out of MANET. Consequently, it can be said that system increases its network scalability to a larger extent as compared to conventional CIDR.

Initially, the mobile nodes that belong to a single the MANET have the possibility of getting partitioned owing to the dynamic topology and lead to multiple small MANET system. Hence, it is important that such critical alterations of the topologies could be evaluated by the specific gateways in all the small group of MANET system. If the system is found to use any proactive routing protocol for establishing communication, then the partition will be involuntarily identified by the routing protocol itself using the concept of periodic route updating. But in case the system is found using reactive routing protocols for partitioning process, the possibility of listening to a control message to go undetected is very high for a moment until a specific control message is sent to a destination node in a disconnected partition. Therefore, in order to meet this demand, SCIDR assumes consideration of gateway for maintaining a transition state of network topological information using regular beacons. If 
these beacons are not properly reached then it leads to partition. In order to overcome such partitioning, SCIDR makes use of active gateways.

The prior techniques of Zhou et al. [19] have considered situation where certain set of mobile nodes are preliminary designated as gateway whose mobility is evaluated by their mission objectives and not by the infrastructure goals However, we strongly feel that this will be serious issue to support higher scalability for performing inter-domain routing in mobile adhoc network. SCIDR principle is based on the concept that it is never wise to consider all the mobile nodes to have chances to be a gateway, which is very much detrimental for design principles of routing in heavy traffic. The critical issue behind this perception is that it will fastly dissipate its energy (battery) even in a situation when the mobile node is not participating in the communication process. Also, there is a higher possibility that it will generate a massive number of control packets leading to unwanted and extra overhead in the network. Therefore, we mitigate the issues of CIDR using following approaches:

SCIDR quantify the energy as well as delay during the computation of inter-domain routing as well as in local distribution stage thereby formulating a multi-variable optimization problem to enhance the performance of routing considering data rate, delay, and energy as a constraints.

SCIDR uses channel state information (CSI) data to identify the best possible condition of routing. The system will choose intermediate nodes for a hop with precise, instantaneous CSI data or channel correlation data.

SCIDR consideration of channel correlation data is fundamentally associated with the shadowing effect in the network. Therefore, we express channel correlation factor as

$$
C C=b t^{\frac{S_{d}}{S_{D}}}
$$

where, bt is a variable representing the correlation between two mobile nodes separated by spatial distance SD and Sd. Sd is the distance between source node and neighbor node while SD is the distance between source node and destination node. SCIDR uses a simple concept of Optimal Channel Gain (OCG). The formulation of OCG is quite simple: at the $(x+1)^{\text {th }}$ step, where the $\mathrm{x}$-mobile nodes have already been selected for routing, the corresponding channel matrix can be designated as hessian metric e.g. H(x). Proposed SCIDR will look for the one extra mobile node $\mathrm{m}^{*}$ from the set of mobile nodes M containing the residual $\mathrm{X}-\mathrm{x}$ mobile nodes, such that

$$
m^{*}=\arg \max _{m^{*} \in M}\left\{\operatorname{det}\left(\left(H^{(x+1)}\right)^{H}\left(H^{(x+1)}\right)\right)\right\}
$$

The entire process is iterated until all the $\mathrm{M}$ mobile nodes are selected. Hence, the system accomplishes various combination of the mobile nodes $\zeta$ with a priority of routing. This can eventually meet the growing demands of the traffic in case of heterogeneous MANET. The algorithm executes for $\mathrm{X}$ rounds and selects best subset $\zeta^{*}$ that results in the largest spatial relationship for the optimal inter-domain routing while satisfying the delay as well as energy constraint.

SCIDR assumes bi-directional pattern of communication between the inter-domain gateways, where domains are already assigned. All the mobile nodes adopt only one type of routing protocol, where the domain may possess multiple clusters that totally depends upon the task allocation schemes.

SCIDR consists of common steps of neighbor maintenance, cluster-based routing, and data exchange among various domains. However, SCIDR doesn't use fisheye scheme for broadcast nor use bloom filters like conventional CIDR protocol. It has its techniques, which is discussed in brief in next section.

\section{DESIGN PRINCIPLE}

The research methodology adopted in the proposed study is more inclined to exploratory approach, as the study intends to formulate a completely novel solution to compensate the research goal. For this purpose, initially a heterogeneous MANET system is formulated where clusters of nodes with one sort of wireless network boundary are gathered mutually with gateway nodes with two or more types of network interfaces for mapping the heterogeneity.

Fig. 1 shows the schematic diagram of such system, where the study intends to design scalable cluster-based inter-domain routing. Such network often adopts long-range radio to connect the cluster of nodes, which in turn use short range high-speed radios to communicate from node to node.
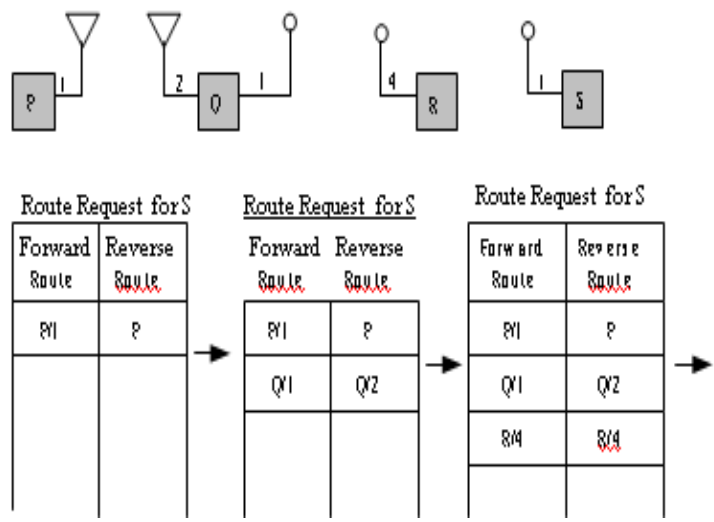

Figure 1: Schematic Diagram for Radio Model for SCIDR

The above schematic diagram shows an example of a MANET with heterogeneous network interfaces. Node P is deploying single type of network interface (shown by the triangles), node $\mathrm{S}$ and node $\mathrm{R}$ are emploing an totally divergent type of physical network interface (shown by the circles), and entrance node $\mathrm{Q}$ is a multi-homed mobile ad-hoc network node that may route among the two dissimilar types of radio technologies. Each node independently chooses an interface index for its interfaces, thus that while Q and $\mathrm{S}$ have mutually elected index 1 for their circle interfaces, $\mathrm{R}$ has selected index 4. It also demonstrates how a route request for $S$ initiates by $P$ will broadcast transversely the network. As the demand propagates it will gather both a forward route from $\mathrm{P}$ to $\mathrm{S}$ and a reverse route from $\mathrm{S}$ to $\mathrm{P}$ (Although every node address is shown twice in each packet, in the concrete packet format used, every address appears only once, together with the interface index for the forward route and the reverse route at each node). When $\mathrm{Q}$ receives $\mathrm{p}^{\text {" }} \mathrm{s}$ route request, $\mathrm{Q}$ checks, if it is previously scheduled on the source route, evidences in the packet or have previously re-propagated a duplicate of this request. If neither is true, $\mathrm{Q}$ adds itself to the listed route and re-propagates the request out all its interfaces, excluding the one it was established on. When Q transmit the packet out interface $\mathrm{i}$, it lists itself in the forward route as $\mathrm{Q} / \mathrm{i}$. R receives the demand and repeats this process, so that when the packet is received at $S$ it contains both a route from $P$ to $S$ and a route from $S$ to $P$. $S$ returns the discovered route, $\mathrm{P} / 1 \rightarrow \mathrm{Q} / 1 \rightarrow$ 
$\mathrm{R} / 4 \rightarrow \mathrm{S}$, to $\mathrm{P}$ in a route reply packet. $\mathrm{S}$ can return the reply to $\mathrm{P}$ using a cached route, using the reverse route collected in the request, or by responsibility route discovery and piggybacking the reply on its request for P. The packet headers in Figure 2 demonstarte how the source route would be employed to route a packet from $\mathrm{P}$ to $\mathrm{S}$, with the outlined boxes representative which hop in the source route is being processed. The source route on a packet as it moves through an ad-hoc network changing physical interface categories from triangle interfaces to circle interfaces.

The outlined boxes signify which entrance in the source route is deployed when transmitting the packet at every stage. This example presents the need for a source route to contain both the home address and interface index of every hop. Otherwise, node $\mathrm{Q}$ would not have the information necessary to determine which of its interfaces should be used when forwarding the packet. Once this information is illustrate in every packet, packets may be routed flawlessly across heterogeneous network interfaces. The control message will travel across the network as it (RREQ) originates from various source routes thereby has better reachability in the given network. Moreover, the control message also effectively propagates in the entire network as each gateway will insert its unique home address into each RREQ message. The proposed system adopts the mechanism of flooding the route request message in the network, which is similar to conventional homogeneous MANET system.

Hence, the target of the proposed study is to integrate different layer 2 devices into one single mobile ad-hoc network, with respect to i) Transparency: Transparent end-to-end communication, ii) Mobility: Common mobility scheme, iii) Addressing: Interface independent addressing, iv) Configuration: Ad-hoc configuration along with scalability. A conceptual model of heterogeneity is shown in figure 3 .

\section{PERFORMANCE PARAMETERS ADOPTED}

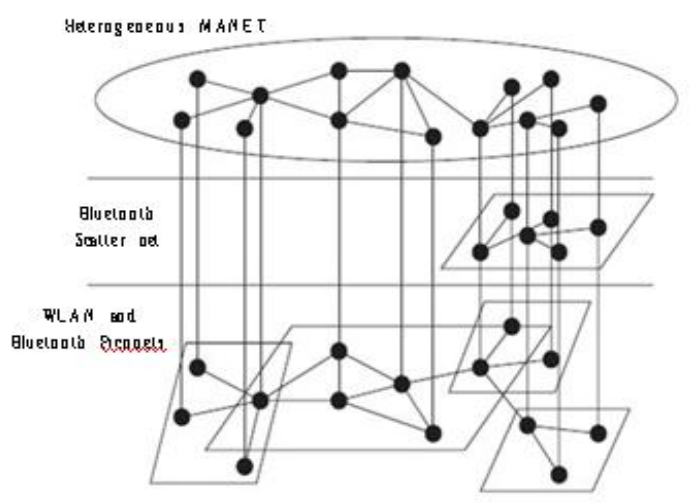

Figure 3: Architectural Model of Heterogeneous Network

Studies from Zhou et al. [19] have considered packet delivery ratio and control overhead only. While designing cluster based inter-domain routing in MANET, these parameters are not enough to infer a much robust conclusion. Hence, for the sake of in-depth investigation, our implementation concept emphasizes on using more discrete parameters for better novelty. Performance parameters considered are as follows:

\subsection{Optimal Channel Gain (OCG)}

The reason for using it: In a MANET, signals usually get from the transmitter to the receiver by some paths, each produced by one or more reflections. Because the paths have various lengths and, therefore, various phase shifts, they might combine constructively or destructively at the receiver. The benefit of using it: Optimal Channel Gain will offer a blond thought about the signal attenuation and phase shift angle patterns, which are highly expected in dynamic mobility scenario of MANET. We expect this parameter will assist better visualization in heterogeneous MANET as it has got the common integration of various network interfaces.

\subsection{Channel State Information (CSI)}

The reason for using it: Scheduling in MANET is already a challenging issue yet to be explored for a better solution [20]. Things become much worst when heterogeneous network interfaces exist in MANET. The benefit of using it: Evaluation of CSI will give a better visualization of topology and channel-state uncertainty in heterogeneous MANET.

\section{MINIMAL CHANNEL CORRELATION (MCC)}

The reason for using it: Channel correlation typically has a significant impact on the performance of a spatial multiplexing in MANET. The best performance of a spatial multiplexing system can be reached when channels are independent. However, MANET channels are often characterized by channel correlations [21].

The benefit of using it: It will only make use of the channel correlation information at the heterogeneous domain and tries to minimize the correlation among the co-operative nodes for better routing.

\subsection{Energy}

The reason for using it: Mobility of nodes with dynamic topology will essentially lead to energy (battery) depletion. As the proposed study focuses on introducing cluster based interdomain routing protocol, it must use energy as performance factor as routing decisions are usually based on minimum energy and interference. The performance evaluation becomes much more challenging when heterogeneous MANET is considered [22]. Benefit of using it: Spontaneous tracking of energy depletion will assist in better routing decision as heterogeneous MANET has various network interfaces (e.g. WSN, WiMax, UMTS, WLAN, etc.), each may have their routing strategy, but RF circuitry design of transmitter is almost same for every node existing in domains.

\subsection{Total Delay}

The reason for using it: In heterogeneous MANET, owing to different node types, their physical layer work differently for different node types, hence possibly lead to network delay. It will also lead the study to understand underlying issues of quality of services. The benefit of using it: To monitor the delay and evaluate the performance of proposed routing protocol.

\subsection{Bit Error Rate - BER}

The reason for using it: In MANET, packet loss- the failure of transmitted packets to reach their destination is evaluated by bit error rate. Wireless links are subject to transmission errors and the dynamic network topology [23]. The benefit of using it: It will give a better idea about the extent of packet loss in proposed framework.

\subsection{Throughput Percentage}

The reason for using it: It helps to understand the fundamental network throughput limit and thus serves as an 
instruction guideline for the network design, performance optimization [24][25]. Benefit of using it: Based on the throughput result, the optimal capacity of SCIDR and its variation to achieve the possible maximum throughput can be evaluated.

\section{RESULT AND DISCUSSION}

The evaluation of the proposed system is done by studying the individual outcomes of the SCIDR as well as comparative performance analysis.

\subsection{Individual Performance Analysis}

For the purpose of evaluating the proposed system, the study considers various critical parameters to understand it e.g. delay, BER performance, and throughput performance under extensive simulation environment.

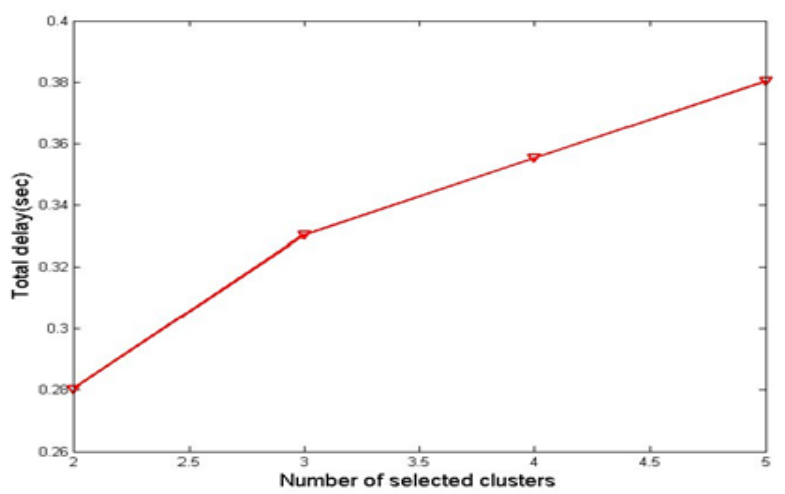

Figure 4: Performance Graph of Clusters vs. Delay

Fig.4 represents the outcome of the delay in seconds. Using probability limits for cumulative network delay, it can be seen that through the increase in number of selected clusters in SCIDR framework, the delay increases. However, a closer look into the delay performance shows only $12 \%$ increment

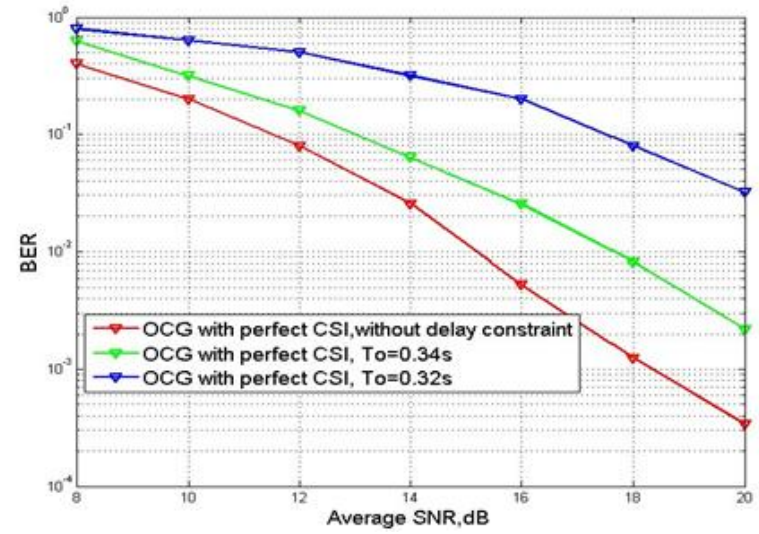

Figure 5: Performance Graph of SNR vs. BER with Varied Delay Constraints of Perfect CSI

approximately in the peak traffic condition. Hence, the system can tolerate number of traffic with significant constraints. Figure.5 exhibits the presentation of the proposed OCG (optimal channel gain) technique adopted in SCIDR with precise information of CSI that is considered as a function to mean SNR (dB) with significant delay constraint. It can be seen in that if there is the absence of delay constraint then overall delay variable could lead to infinite value, where the optimal performance of the system could be accomplished. However, the system performance tends to decline drastically if the delay constraint becomes hard threshold. The outcome shows that proposed technique of SCIDR using OCG with perfect CSI considering Time slots of $0.34 \mathrm{sec}$ and $0.32 \mathrm{sec}$ assumed hypothetically has considerably higher BER compared to that of without delay constraint. It should be noted that this analysis is done considering energy and time as constraint factors, as in heterogeneous MANET system, selection of best set of mobile nodes for performance enhancement is not always possible owing to dynamic topology and so constraints of energy and time plays a critical role.

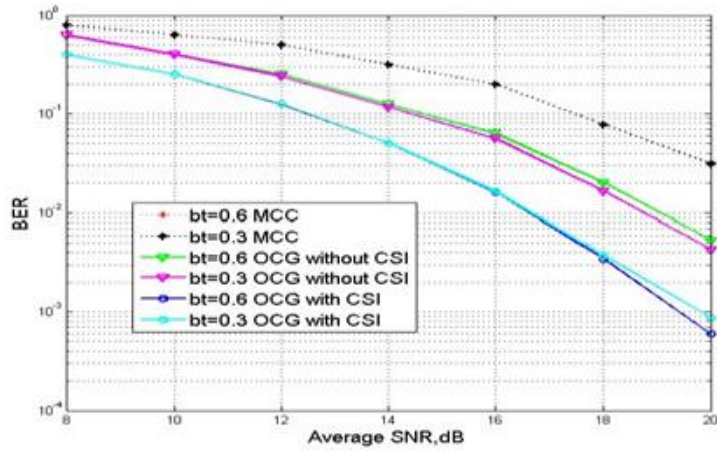

Figure 6: Performance Graph of SNR vs. BER for Varied bt Conditions

Fig.6 exhibits the outcome of the proposed SCIDR under numerous channel correlation conditions. The outcome shows that proposed SCIDR along with OCG technique can have better performance with the inclusion of perfect CSI data in each cluster as it can use it for selection of the best mobile node for performing heterogeneous communication. The proposed analysis initiates by assuming value of variable bt (correlation between two mobile nodes) as 0.3, however, it was altered under different condition of proposed system with and without consideration of CSI to visualize the performance. The system also considers MCC (Minimal Channel Correlation) for analysis, which doesn't show better BER performance. Optimal BER performance could be seen only when the beta value is either 0.3 or 0.6 using OCG with CSI. Hence, CSI plays a crucial role in mitigating the impediments towards communication in heterogeneous MANET system. A closer look at the outcome also shows that it is quite possible to implement SCIDR in situation when CSI is not available in the network, and hence channel correlation factor becomes a very good alternative to substitution of CSI factor. This fact can be established if the proposed SCIDR enhances OCG technique without CSI. A unique pattern is observed in the curves which shows performance degradation with increase of channel correlation.

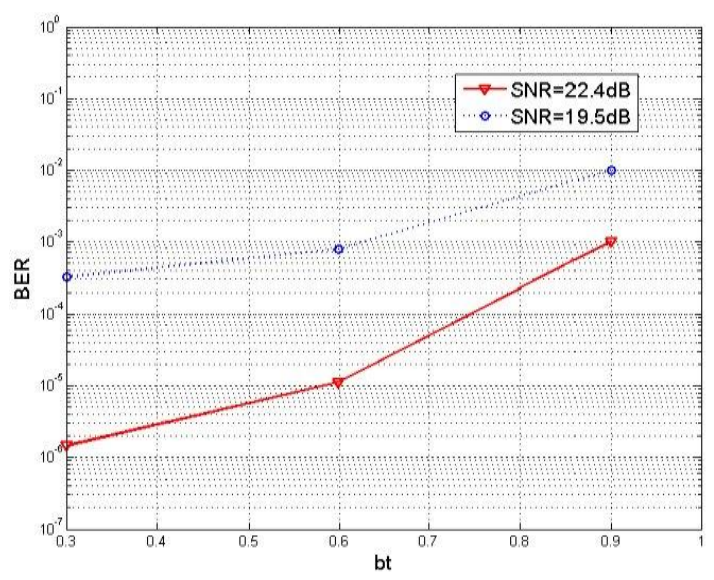

Figure 7: Performance Graph of BT vs BER for Varied SNR Values 
Fig.7 shows the BER performance under two different SNR condition and with increasing value of channel correlation factor bt. From Fig.6, it was quite evident that channel correlation factor bt could be adopted in the case when CSI is absent, and hence this part of the evaluation performs analysis of it. The outcome shows that with an increase of channel correlation in every cluster, the degradation in system performance is observed. The outcome also indirectly highlights that CSI is a better option compared to channel correlation factor exclusively in heterogeneous MANET system.

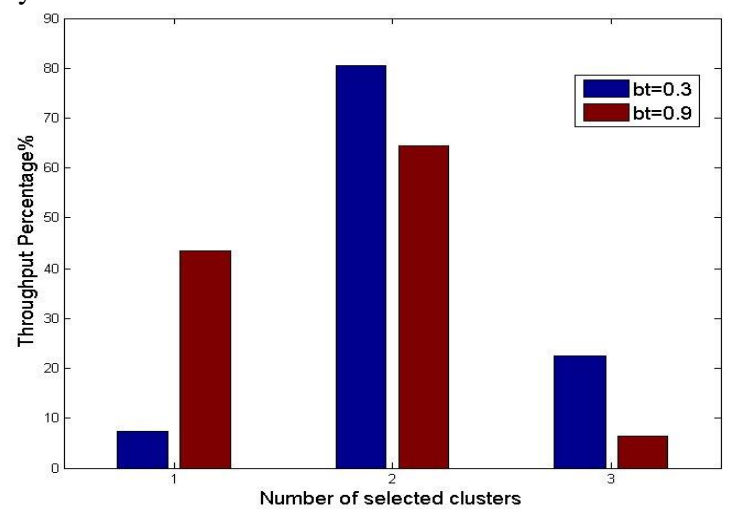

Figure 8: Performance Graph of Cluster vs. Throughput for. Varied bt Values

Fig.8 represents the outcome of the throughput percentage with 3 test clusters. The evaluation is performed using two different channel correlation factor (bt $=0.3$ and 0.9 ). The outcome shows that when the channel correlation increases, throughput minimizes. Hence, for better throughput, channel correlation factor should be kept as less as possible to overcome the control overhead and data redundancy which is very frequent in heterogeneous MANET system

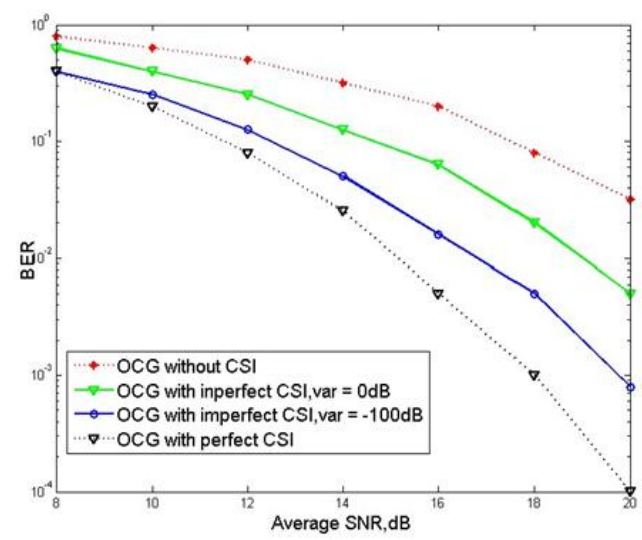

Figure 9: Performance Graph of Avg SNR vs. BER with Varied Conditions of OCG

Fig.9 exhibits the extended analysis of BER performance with respect to SNR under multiple channel condition and error in channel estimation, which is very much frequent in interdomain routing in heterogeneous MANET system. The system adopts sophisticated Gaussian arbitrary variable with zero means as the error in channel estimation owing to node mobility as well as acknowledgment delay in the routing process. We consider the statistically evaluated CSI data to be a summation of a random variable with zero mean, variance, and actual CSI. The outcome shows that proposed system SCIDR with OCG technique and precise CSI data accomplishes better BER performance with an increase of channel estimation error (SNR, dB). The outcome essentially shows that channel correlation factor is the better option for the scenario with large error probability in channel estimation. In such scenario, CSI is not a better solution.

\subsection{Comparative Performance Analysis}

For the purpose of comparative analysis, we choose the standard work done by Zhou et al. [19] who have introduced actual CIDR protocol for performing inter-domain routing in MANET system. The CIDR protocol has also targeted to solve the scalability issues of MANET by adopting cluster based routing using border gateway protocol. The protocol was designed using fisheye scheme for performing broadcasting and bloom filters for membership management. The final analysis of CIDR was done using packet delivery ratio and control overhead.

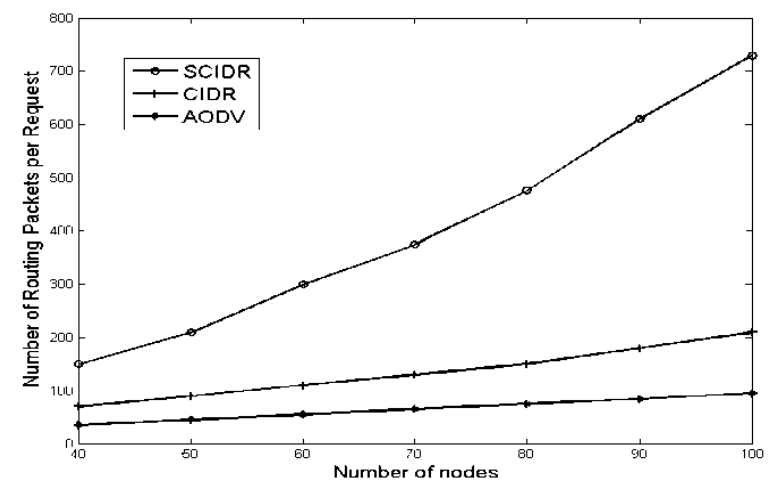

Figure 10: Routing Packets per Request

We design SCIDR as the enhanced version of CIDR protocol, where, the previous section have performed extended analysis considering multiple sophisticated network scenarios. These sections will explicitly analyze the outcome of proposed SCIDR with conventional CIDR to perform benchmarking. Fig.10 shows the outcome routing packet per request with the increasing number of the mobile nodes. To demonstrate the comparative performance, analytical data is taken for CIDR and AODV from various respective articles and for SCIDR, average simulation result is considered. The outcome shows that AODV had higher routing overhead as compared to conventional CIDR and proposed SCIDR. Owing to this outcome, it is quite eventual that route set time considered in heterogeneous MANET system does have similar performance. The proposed system of SCIDR does show much better performance of scalability and reduction in routing overhead as compared to conventional CIDR $(\mathrm{O}(\mathrm{n} 2)$, where $\mathrm{n}$ is a number of nodes).

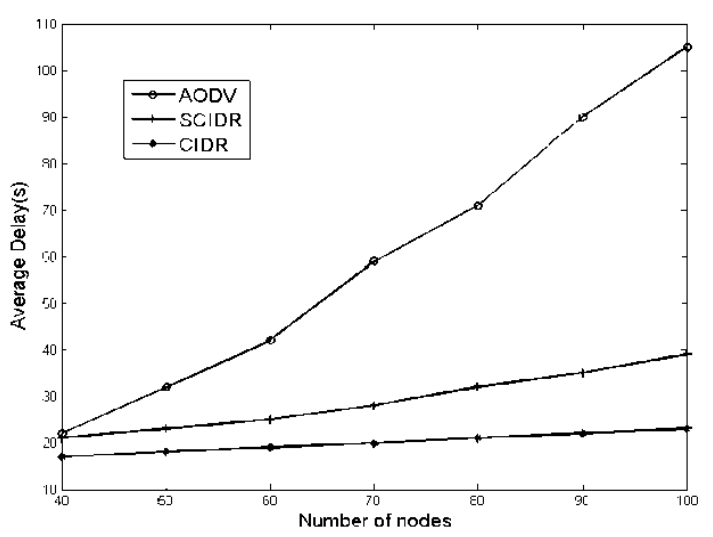

Figure 11: Performance Graph of No. of Nodes vs. Average Delay for AODV, SCID and CIDR 
Fig.11 discusses the average delay of the proposed SCIDR with conventional CIDR as well as AODV. The frequent adoption of routing protocol AODV in MANET was mainly targeted to minimize the delay and enhance the packet delivery rate. Hence, the average delay of the AODV is found significantly higher. To a large extent, usage of conventional CIDR has better performance compared to AODV, but owing to the adoption of BGP protocol, such delay constraints couldn' t be optimally satisfied. However, proposed SCIDR is built on the top of enhanced version of CIDR by incorporating various parameters that can understand the CSI as well as channel correlation factor based on inter-domain routing principle in heterogeneous MANET, for which reason, the delay performance is found superior even compared to conventional CIDR. Fig.12 shows the comparative performance analysis with respect to energy consumption as energy and time are one of the considered constraints involved in our study design principles. The evaluation is carried out by computing cumulative energy consumption divided by the cumulative number of packets received. It is said that if the routing is carried out more effectively in dynamic topology like MANET, a higher extent of the energy of the node could be preserved.

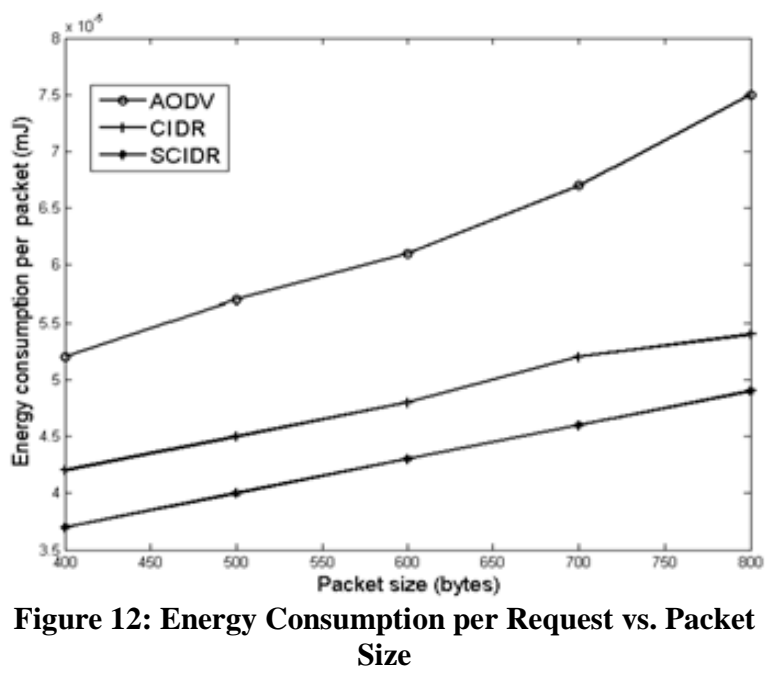

The outcome shows that widely used AODV routing protocol is found to have the poor performance of energy conservation while performing data transmission in MANET. However, AODV is not able to cater up the demands of inter-domain routing in heterogeneous MANET, for which reason, energy performance degrades significantly. One of the interesting fact explored in this part of the analysis is that both conventional CIDR as well as proposed SCIDR protocols performs search of path that are energy efficient in spite of choosing the path with shortest distance, as seen in AODV. The prior outcomes (Fig.11) have already exhibited that CIDR has poor delay performance which means routing overhead is quite higher. Unfortunately, the request for the inter-domain route search using CIDR was found to have maximum probability of volatile route that are energy efficient by the intermediate mobile node itself. For which reason, neither the scalability nor the route stability can be achieved using conventional CIDR protocol. This is one of the prime reasons for which both CIDR as well as SCIDR performs better than AODV. Proposed SCIDR explores the better inter-domain routes with better cost along with CSI and channel correlational factor for which reason SCIDR can choose the path with better stability in energy depletion.

Fig.13 represents the energy consumption with respect to the node velocity. A mobile node while travelling on random or specific velocity depletes maximum quantity of energy. The problem of energy conservation is homogenous MANET system is continually being addressed by research community from last few decades, but the situation is completely different when heterogeneous MANET system is considered.

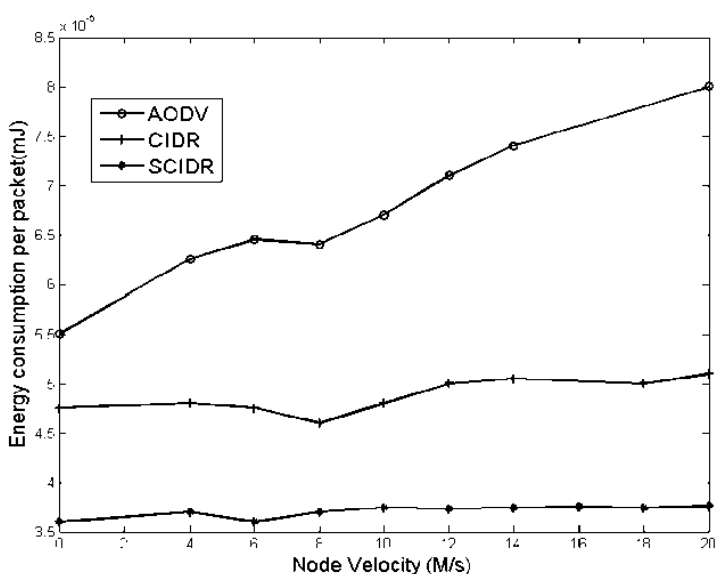

Figure 13: Effect of Energy Consumption on Varied Node Velocity

As in heterogeneous MANET system, the mobile devices are of a different hardware configuration for which reason the rate of energy depletion will differ from one to another mobile node. AODV performance is found to be quite declining in nature as it has the poor performance of end-to-end delay. Another issue of performance degradation of AODV is because of formation of inconsistent routes with stale entries of routes leading to maximized control overhead and unwanted bandwidth consumption for the mobile nodes that finally leads to faster degradation of energy factor with increasing node velocity. CIDR protocol is found to have better performance compared to AODV with increasing node velocity. However, one of the issues of CIDR is that it adopts bloom filter for overcoming the issues of membership management in inter-domain routing of MANET. As Bloom filters have the inclusion of false positives in its outcome, hence, the reliability of the routing performance cannot be purely justified in the case of heterogeneous MANET system. The proposed SCIDR overcomes this problem by using interior gateway protocol and using effective channel based parameters required for performing inter-domain routing using CSI, which can capture better information of multiple different domains of MANET system. Hence, better energy mitigation solution could be furnished by proposed SCIDR protocol. Hence, the proposed system could effectively address the scalability parameters.

\section{CONCLUSION}

The proposed work is attempted to redefine the concept of scalability in heterogeneous MANET. The evidence to prove it is that the prior authors have associated scalability with numbers of mobile nodes, where packet delivery ratio, throughput, latency, delay, inter-arrival time, etc. are the common parameters to evaluate scalability. However, an indepth investigation on prior studies have also witnessed that existing inter-domain routing techniques have never adopted channel parameters (CSI, MCC, OCG, Energy) to evaluate scalability. It is well known that there is higher extent of issues like scattering, fading, noise in the network that is also applicable in heterogeneous MANET. Hence, the proposed system has introduced a new routing protocol that ensures scalability in maximum respect to guaranteeing superior 
system and network performance of inter-domain routing in heterogeneous MANET. The proposed system has presented a new scheme called as SCIDR, which is the enhanced version of conventional routing standard CIDR. The design principle is based on some near-real-time assumptions that emphasizes on considering energy and end-to-end delay as the constraint. With the support of simple optimal channel gain algorithm, the proposed SCIDR became much cost effective and gave higher range of scalability to perform inter-domain routing. One of the uniqueness of this paper is that for the first time the inter-domain routing protocol is validated with the extensive performance parameters that were not seen in any studies of heterogeneous MANET system in the past literature. Our simulation result being benchmarked with conventional CIDR protocol to find that SCIDR protocol can support higher dimensionality of scalability. Also it can be benchmarked to various other qualities of service parameters even for peak traffic condition to perform inter-domain routing in heterogeneous MANET system.

\section{REFERENCES}

[1] Basagni, S., Conti, M., Giordano, S., Stojmenovic, I. (2013). Mobile Ad Hoc Networking: The Cutting Edge Directions, John Wiley \& Sons, Technology \& Engineering, pp. 888

[2] Singh, G., Singh, J.(2012).MANET: Issues and Behavior Analysis of Routing Protocols, “ International Journal of Advanced Research in Computer Science and Software Engineering", Vol. 2, Iss.4

[3] Beijnum, I. (2002).BGP: Building Reliable Networks with the Border Gateway Protocol, O'Reilly Media, Inc, Computers, pp. 290

[4] Chau, C-K., Crowcroft, J., Lee, K-W., Wong, SH.K. (2008). Inter-Domain Routing for Mobile Adhoc Networks, ACM-MobiArch

[5] Rekha, B., Ashoka, D.V. (2013).An Enhanced InterDomain Communication among MANETs through selected Gateways, Int. J. on Recent Trends in Engineering and Technology, Vol. 9, No. 1

[6] Chuah, M.C., Yang, P. (2014). Performance Comparison of Two Inter-domain Routing Schemes for Disruption Tolerant Networks, Cite Seer

[7] Dressler, F., Gerla, M. (2013). A framework for interdomain routing in virtual coordinate based mobile networks, Wireless Networks, Vol. 19, No. 7, pp. 16111626

[8] Dressler, F., Koch, R., Gerla, M. (2012). Path heuristics using ACO for inter-domain routing in mobile ad hoc and sensor networks, In Bio-inspired models of network, information, and computing systems, pp. 128-142

[9] Okundaye, I.(2013).Inter-domain Routing for Tactical Mobile Ad-hoc Networks, Master of Applied Science in Electrical and Computer Engineering, Carleton University

[10] Comarela, G., Gürsun, G., and Crovella, M.(2013).Studying inter-domain routing over long timescales, In Proceedings of the conference on Internet measurement conference, pp. 227-234

[11] Kaur, B., Singh, K., Sharma, S. (2013). Inter-Domain Routing with Shielded Infrastructure and Buzzer
Technique, International Journal of Computer Applications, Vol. 74, No.15

[12] Elmokashfi, A., Kvalbein, A., and Dovrolis, C. (2011). „Simrot: a scalable inter-domain routing toolbox, ACM SIGMETRICS Performance Evaluation Review, Vol. 39, No. 2, pp. 4-13

[13] Yuanling, L., Jinjing, Z., Li, L., Peidong, Z. (2014). „Building a Threat Model of IPv6 Inter-domain Routing System, Recent Advances in Telecommunications and Circuit Design

[14] Pan, J., Jain, R., and Paul, S. (2013). „Enhanced Evaluation of the Interdomain Routing System for Balanced Routing Scalability and New Internet Architecture Deployments, IEEE Systems Journal

[15] Wang, Y., Bi, J., Lin, P. (2014). SRP: Building a Software Defined Inter-domain Routing Plane via SDN

[16] He, Y., Faloutsos, M., Krishnamurthy, S.V., Chrobak, M.(2008). Policy-Aware Topologies for Efficient InterDomain Routing Evaluations, in Proc. INFOCOM, pp. $2342-2350$

[17] Lee, S-H., Wong, S.H.Y., Chau, C-K., Lee, K-W., Crowcroft, J., Gerla, M.(2010). In terms: Inter-manet routing in heterogeneous manets, In Mobile Adhoc and Sensor Systems (MASS), IEEE 7th International Conference, pp. 372-381

[18] Javed,U., Cunha, I., Choffnes, D.R., Bassett, E.K., Anderson, T., Krishnamurthy, A.(2013).PoiRoot: Investigating the root cause of interdomain path changes, In Proceedings of the ACM SIGCOMMconference on SIGCOMM, pp. 183-194

[19] Zhou, B., cao, Z., Gerla, M.(2009).Cluster-based Interdomain Routing (CIDR) Protocol for MANET ${ }^{e e}$, IEEE

[20] Ying, L., Shakkottai, S.(2012). Scheduling in Mobile Ad Hoc Networks With Topology and Channel-State Uncertainty, IEEE Transactions on Automatic Control, Vol. 57, No. 10

[21] Xie, M., Haenggi, M.(2007). A Study of the Correlations between Channel and Traffic Statistics in Multihop Networks, IEEE Transactions on Vehicular Technology, Vol. 56, No. 6

[22] Cavalcanti, D., Agrawal, D., Cordeiro, C., Xie, B., Kumar, A.(2005). Issues in integrating cellular networks WLANs, AND MANETs: a futuristic heterogeneous wireless network ${ }^{e e}$, Wireless Communications, Vol. 12 No. 3, pp.30-41, 2005

[23] Yadav, J., Garg, N., Sharma, N. (2013). Analysis of Packet loss and Throughput in Heterogeneous Mobile Ad-hoc Networks over UDP, International Journal of Scientific \& Engineering Research, Vol.4, Iss. 6

[24] Vidhya, S., Sahaya, G., Jose, S.(2014). Increased Throughput in MANETS with the Heterogeneous Environmente, International Journal of Technology Enhancements and Emerging Engineering Research, Vol.2, Iss.4

[25] Pillai, M.J., Sebastian, M.P.(2009).Improving Energy Efficiency and Throughput in Heterogeneous Mobile Ad Hoc Networks, International Journal of Mobile Computing and Multimedia Communications (IJMCMC), Vol. 1, No. 2, pp. 48-60. 